Romankunst in Latcinamerika 
Der moderne lateinamerikanische Roman ist nicht nur Gegenstand internationalen Interesses, sondern nimmt zunehmend Einfluß auf den weltliterarischen Prozeß. $\mathrm{Da}$ sich die lateinamerikanische Prosa relativ spät entwickelte, übernahm sie auch Elemente der Literaturen anderer Völker und vergangener Zeiten. So wurden Joyce, Kafka, Dos Passos und der Nouveau Roman ebenso integriert wie die Formen des höfischen, des Ritter- und Schelmenromans. Daraus ergibt sich eine originelle Verbindung verschiedener Traditionen und Methoden. Mythos und Wirklichkeit, Dokumentarisches und Phantastisches verschmelzen in dieser Literatur zu einem organischen Ganzen.

Dieser Studienband führt ein in die Entwicklung des modernen Romans in Lateinamerika. Am Beispiel von zwölf repräsentativen Autoren - Klassikern der zeitgenössischen Prosa und weniger bekannten, jüngeren Romanciers - werden die Wechselwirkung zwischen Literatur, Gesellschaft und Geschichte, literarische Traditionen, ästhetische Strukturen und Wirkungsstrategien untersucht. Auf voreilige Kategorisierungen und Theoriebildungen wird verzichtet. Anliegen der Studien ist es vielmehr, "die jeweils neuen literarischen Lösungen zu verdeutlichen, die der Roman für die Probleme fand, die sich ihm im Fortgang der Geschichte stellten" (Manfred Naumann).

\section{Hermann Herlingbaus}

1954 geboren; Studium der Lateinamerikawissenschaften an der Wilhelm-PieckUniversität Rostock; Promotion 1985; Studienreisen nach Kuba, Spanien, Chile und den USA; seit 1986 am Zentralinstitut für Literaturgeschichte der Akademie der Wissenschaften der DDR; Kritiken, Aufsätze und Essays zur zeitgenössischen Literatur- und Filmentwicklung in Lateinamerika. 


\section{Romankunst \\ in Lateinamerika}

Herausgegeben

von Hermann Herlingbaus

Akademie-Verlag Berlin 1989 


\section{Fotonacbweis}

Prensa Latina (Jorge Luis Borges, Julio Cortázar, Gabriel Garcia Márquez, Manuel Cofiño) ADN-ZB/AFP (Miguel Angel Asturias)

ADN-ZP/Prensa Latina (Alejo Carpentier)

Roberto Riquenes (Antonio Skármeta)

Pildain (Manuel Rojas)

Archiv Herlinghaus (Mario Vargas Llosa, Carlos Fuentes, Jorge Amado, Isabel Allende)

ISBN 3-05-000637-4

Erschienen im Akademie-Verlag Berlin, DDR - 1086 Berlin, Leipziger Str. 3-4

(c) Akademie-Verlag Berlin 1989

Lizenznummer: $202 \cdot 100 / 112 / 88$

Printed in the German Democratic Republic

Gesamtherstellung: VEB Druckerei „Gottfried Wilhelm Leibniz",

4450 Gräfenhainichen $\cdot 7017$

Lektor: Alfred Gessler

Einbandgestaltung: Ralf Michaelis nach einer Grafik von Lorena Araya Valenzuela (Santiago de Chile)

LSV: 8061

Bestellnummer: 7548823 (9107)

02800 